\title{
Well-posedness and exponential stability of the Kawahara equation with a time-delayed localized damping
}

\author{
Boumediène Chentouf ${ }^{1}$ \\ ${ }^{1}$ Kuwait University
}

January 17,2022

\begin{abstract}
The aim of this article is to investigate the well-posedness and stability problems of the so-called Kawahara equation under the presence of an interior delayed damping. The system is shown to be well-posed. Furthermore, we prove that the trivial solution is exponentially stable in spite of the delay effect. Specifically, local and semi-global stability results are established according to the properties of the spatial distribution of the delay term.
\end{abstract}

\section{Hosted file}

kawa int delay.pdf available at https://authorea.com/users/455750/articles/553028-wellposedness-and-exponential-stability-of-the-kawahara-equation-with-a-time-delayedlocalized-damping 